\title{
Far-infrared edge modes in quantum dots
}

\author{
E. Lipparini,* N. Barberán, M. Barranco, and M. Pi \\ Departament d'Estructura i Constituents de la Matèria, Facultat de Física, Universitat de Barcelona, E-08028 Barcelona, Spain \\ L1. Serra \\ Departament de Física, Facultat de Ciències, Universitat de les Illes Balears, E-07071 Palma de Mallorca, Spain
}

(Received 14 May 1997)

\begin{abstract}
We have investigated edge modes of different multipolarity sustained by quantum dots submitted to external magnetic fields. We present a microscopic description based on a variational solution of the equation of motion for any axially symmetric confining potential and multipole mode. Numerical results for dots with different numbers of electrons, whose ground state is described within a local current density-functional theory, are discussed. Two sum rules, which are exact within this theory, are derived. In the limit of a large neutral dot at $B=0$, we show that the classical hydrodynamic dispersion law for edge waves $\omega(q) \sim \sqrt{q \ln \left(q_{0} / q\right)}$ holds when quantum and finite-size effects are taken into account. [S0163-1829(97)01644-5]
\end{abstract}

\section{INTRODUCTION}

Collective excitations induced in finite fermion systems by external probes have been extensively studied in the last years. Particular effort has been devoted to an understanding of the giant dipole resonance in nuclei, ${ }^{1}$ and of the plasmon mode in metallic clusters. ${ }^{2-5}$ Recently, a strong collective state has also been observed in quantum dot structures. ${ }^{6,7}$ These collective modes all have in common the feature of being $L=1, S=0$ excited states induced by a dipolar external radiation which is the dominant component of the electromagnetic field when its wavelength is much larger than the size of the system. Dipole modes correspond to oscillations of protons against neutrons in nuclei, and of electrons against the positive background in metal clusters and dot structures. Whereas in nuclei the restoring force of the collective motion is the symmetry potential which acts differently on protons than on neutrons, in metal clusters and dots it arises from the Coulomb interaction between ions and electrons.

Collective states with multipolarity $L>1$ were predicted to exist, and have been observed in nuclei. At present, we have a complete systematics of quadrupole $(L=2)$ and octupole $(L=3)$ collective excitations in nuclei. ${ }^{1,8,9}$ Multipole collective states in ${ }^{3} \mathrm{He}$ droplets have also been studied, ${ }^{10-12}$ but have not been experimentally detected so far. In metal clusters, the predicted multipole states ${ }^{13,14}$ also have not been observed. The basic reason is the experimental difficulties arising from the fact that free clusters are produced and analyzed on-fly, with the added difficulty in the case of ${ }^{3} \mathrm{He}$ drops of being electrically neutral systems.

Far-infrared absorption spectroscopy experiments on large radius quantum dots submitted to a static external magnetic field $B$ have likely evidenced quadrupole excitations, ${ }^{7}$ and an anticrossing between the $L=1$ and 2 resonances, each of them split into two branches, one with negative and another with positive $B$ dispersion. We recall that, for these systems, $L$ has to be understood as the angular momentum about an axis perpendicular to the dot plane.

The collective spectrum of quantum dots has been ad- dressed in different ways. An explanation based on classical arguments can be found in Ref. 7 In Refs. 15-18, use has been made of an edge-magnetoplasmon model, whereas in Refs. 19 and 20 a Hartree random-phase approximation (RPA) method has been employed, and a Hartree-Fock RPA one in Ref. 21. Multipole modes have been considered in Refs. 15 and 20. All these approaches neglect the electron correlation energy, and therefore, no microscopic calculation of $L \geqslant 1$ modes exists which incorporates exchange correlation energy as well as quantum effects arising from finite size and shell structure of dots in a magnetic field. A workable, yet reliable formalism that takes into account all these effects is called for to describe these excitations. In this paper we present one of such possible formalisms. It is based on the equation of motion method within the framework of a local current density-functional theory (CDFT) ${ }^{22}$ This functional theory is well suited to study electronic systems in presence of a magnetic field, and has been successfully employed to study ground-state (g.s.) properties of quantum dots. ${ }^{23}$

This paper is organized as follows. We describe the equation of motion method in Sec. II, as well as the basics of the strength function and its moments (sum rules) needed to interpret the experimental and theoretical results. In Sec. III we present a rigorous solution of the dipole mode in the case of the full Pauli many-electron Hamiltonian with a parabolic lateral confining potential. The exactness of the dipole solution in this case, irrespective of the value of the magnetic field, has been discussed in Refs. 24 and 25. Here we have obtained this result in a way that yields an explicit expresion not only for the spectrum, but also for the eigenstates. In Sec. IV we present a variational approach to the description of multipole excitations in which exchange and correlation effects are taken into account within CDFT. We show in Sec. $\mathrm{V}$ that for neutral large dots at $B=0$, these excitacions build an edge wave with dispersion relation of the type $\omega(q) \sim \sqrt{q \ln \left(q_{0} / q\right)}$. Detailed numerical results are presented in Sec. VI for dots with different number of electrons, and the concluding remarks are presented in Sec. VII. Finally, an appendix contains some technical details. 


\section{EQUATION-OF-MOTION METHOD}

We consider $N$ electrons moving in the $z=0$ plane where they are confined by the dot potential $V_{+}(\mathrm{r})$ with $r=\sqrt{x^{2}+y^{2}}$. On this system it may act a constant magnetic field in the $z$ direction described by the vector potential $\vec{A}=1 / 2(-y, x, 0) B$, and we suppose that the system can be described by the $N$-electron Pauli Hamiltonian:

$$
\begin{aligned}
H= & \sum_{i=1}^{N}\left\{\frac{1}{2 m^{*}}\left[\vec{p}_{i}+\frac{e}{c} \vec{A}\left(\vec{r}_{i}\right)\right]^{2}+V_{+}\left(r_{i}\right)+\frac{1}{2} g^{*} \mu_{B}^{*} \overrightarrow{\sigma_{i}} \cdot \vec{B}\right\} \\
& +\sum_{i<j}^{N} V_{c}\left(\left|\vec{r}_{i}-\vec{r}_{j}\right|\right)
\end{aligned}
$$

where $m^{*}$ is the electron effective mass which together with a dielectric constant $\epsilon$ and gyromagnetic factor $g^{*}$ are characteristics of the semiconductor (for example, $g^{*}=-0.44$, $\epsilon=12.4$, and $m^{*}=0.067 m_{e}$ in GaAs), $\mu_{B}^{*}$ is the effective Bohr magneton $\mu_{B}^{*}=\hbar e /\left(2 m^{*} c\right), \vec{\sigma}$ is the Pauli matrix vector, and $V_{c}$ is the electron-electron $(e-e)$ interaction

$$
V_{c}\left(\left|\vec{r}_{i}-\vec{r}_{j}\right|\right)=\frac{e^{2}}{\epsilon} \frac{1}{\left|\vec{r}_{i}-\vec{r}_{j}\right|} .
$$

Equation (1) can be rewritten as

$$
\begin{aligned}
H= & \sum_{i=1}^{N}\left\{\frac{\vec{p}_{i}^{2}}{2 m^{*}}+\frac{1}{2} \omega_{c} l_{z_{i}}+\frac{1}{8} m^{*} \omega_{c}^{2} r_{i}^{2}+\frac{1}{2} g^{*} \mu_{B}^{*} B \sigma_{z_{i}}\right. \\
& \left.+V_{+}\left(r_{i}\right)\right\}+\sum_{i<j}^{N} V_{c}\left(\left|\vec{r}_{i}-\vec{r}_{j}\right|\right),
\end{aligned}
$$

where $\omega_{c}=e B / m^{*} c$ is the cyclotron frequency and $l_{z_{i}}$ is the angular momentum operator about the $z$ axis:

$$
l_{z}=-i \hbar \frac{\partial}{\partial \theta} .
$$

Given the exact g.s. $|0\rangle$ of the $N$-electron system, it is possible to obtain the exact spectrum corresponding to a broad class of collective vibrations if one is able to find an operator $\mathrm{O}^{+}$such that the following equation of motion is fulfilled:

$$
\left[H, O^{+}\right]=\hbar \omega O^{+} \text {. }
$$

The state $O^{+}|0\rangle$ has an excitation energy $\hbar \omega$, and the g.s. fulfills $O|0\rangle=0$. As the excited states have a well-defined angular momentum, so the operators $\mathrm{O}^{+}$must have. Consequently, one has to solve Eq. (5) for each $L$ value.

When a magnetic field acts perpendicularly on the dot, it causes a splitting of the excited $B=0$ states into two branches of energy $\hbar \omega_{ \pm L}$, each carrying an angular momemtum $\pm \hbar L$ over that of the g.s. It implies that, besides Eq. (5), the physically acceptable $O_{ \pm L}^{+}$operators have to fulfill

$$
\left[L_{z}, O_{ \pm L}^{+}\right]= \pm \hbar L O_{ \pm L}^{+}
$$

with $L=1,2,3, \ldots$ and $L_{z}=\sum_{i=1}^{N} l_{z_{i}}$. If $\hbar L_{0}$ is the angular momentum of $|0\rangle$, the states $| \pm L\rangle \equiv O_{ \pm L}^{+}|0\rangle$ have an angular momentum $\hbar\left(L_{0} \pm L\right)$.

To ease the formulas that otherwise would be very cumbersome, from now on we shall be using effective atomic units, defined by $\hbar=e^{2} / \epsilon=m^{*}=1$. In this system of units, the length unit is the Bohr radius $a_{0}$ times $\epsilon / m^{*}$, and the energy unit is the Hartree times $m^{*} / \epsilon^{2}$, which we call $a_{0}^{*}$ and $E_{H}^{*}$, respectively. For GaAs we have $a_{0}^{*} \sim 97.94 \AA$ and $E_{H}^{*} \sim 11.86 \mathrm{meV}$.

It is obvious that to find these operators is in general as difficult a task as to solve the Schrödinger equation corresponding to the Hamiltonian Eq. (1) for the vibrational states, and one is led to solve Eq. (5) in an approximate way, one of these being, for example, the random-phase approximation. Another fruitful approximation, originally proposed by Feynman to describe density excitations of superfluid ${ }^{4} \mathrm{He},{ }^{26}$ consists of making an ansatz on $O^{+}|0\rangle$. Acting upon $|0\rangle$ with Eq. (5) and projecting onto $O^{+}|0\rangle$, one obtains

$$
\omega=\frac{\left\langle 0\left|\left[O,\left[H, O^{+}\right]\right]\right| 0\right\rangle}{\left\langle 0\left|\left[O, O^{+}\right]\right| 0\right\rangle} .
$$

Equations (5) and (7) are completely equivalent if $|0\rangle$ is the exact ground state, and $\mathrm{O}^{+}$is the sought operator. The advantage of Eq. (7) is that we may look for approximate solutions of variational type, guessing $\mathrm{O}^{+}$and obtaining $|0\rangle$ within a workable, yet accurate scheme, such as the localdensity approximation (LDA) at $B=0$, or CDFT at $B \neq 0$. To find these approximate solutions is the subject of Sec. IV. We shall also see that, remarkably, Eqs. (1) and (7) have exact solutions in the dipole case when the confining potential has a parabolic form.

The excitation spectrum of the system is usually probed by different external fields, or given the well-defined angular momentum of the excited states, by a selected $L$-polar component of the field. For an excitation operator $F$ representing it, a useful, often experimentally accessible quantity, is the so-called strength function

$$
S(E)=\sum_{n}|\langle n|F| 0\rangle|^{2} \delta\left(E-E_{n}\right)
$$

where $E_{n}$ and $|n\rangle$ are, respectively, the excitation energy and the excited state, and the sum or integral in the case of continuum spectrum extends over all excited states of the system. Of special interest are some energy moments of the strength function

$$
m_{k}=\int E^{k} S(E)=\sum_{n} E_{n}^{k}|\langle n|F| 0\rangle|^{2}
$$

which we shall call sum rules (SR's). They are the $m_{1}$ and $m_{3}$ moments, which can be also written as

$$
m_{1}=\frac{1}{2}\left\langle 0\left|\left[F^{+},[H, F]\right]\right| 0\right\rangle
$$

$$
m_{3}=\frac{1}{2}\left\langle 0\left|\left[\left[H,\left[H, F^{+}\right]\right],[H, F]\right]\right| 0\right\rangle .
$$


These SR's have been extensively studied in the literature. ${ }^{1,8}$ For the present purposes it is enough to recall that, if only one excited state is contributing to $S(E), E_{3} \equiv\left(m_{3} / m_{1}\right)^{12}$ coincides with the corresponding excitation energy. In a more physical situation, whenever the strength is concentrated in a narrow, "high" -energy region, $E_{3}$ is a fair approximation to the resonant energy. We call $E_{3}$ the scaling energy because $m_{3}$ can also be obtained by scaling the $|0\rangle$ wave function as

$$
|\eta\rangle=e^{\eta[H, F]}|0\rangle,
$$

and then carrying out the following derivative:

$$
m_{3}=\left.\frac{1}{2} \frac{\partial^{2}}{\partial \eta^{2}}\langle\eta|H| \eta\rangle\right|_{\eta=0} .
$$

The $m_{3}$ sum rule can be obtained either from Eq. (10) or (12). The latter allows one to identify $m_{3}$ as the restoring force that causes the collective vibration, and, consequently, to identify $m_{1}$ with the collective mass parameter.

We want to stress that not all possible approximation schemes fulfill the $m_{1}$ and $m_{3}$ sum rules in the sense that a direct evaluation of Eq. (9) yields the same result as Eqs. (10). A test on the consistency of the approximations made to get the g.s. and excitation spectrum, is the fulfillment of these SR's, especially of $m_{1}$, which is very model independent.

\section{EXACT SOLUTION OF THE DIPOLE MODE FOR THE PARABOLIC CONFINING POTENTIAL}

It has been frequently argued in the literature that $V_{+}(r)=1 / 2 \omega_{0}^{2} r^{2}$ can be a good approximation of the confining potential. That is the case, for example, when the number of electrons in the dot is small as compared with the number of positive ions $N_{+}$that produce the confining potential. ${ }^{27}$ If that approximation holds, it is easy to check that the solutions of Eq. (7) for the Hamiltonian Eq. (3) and $L=1$ are

$$
\begin{gathered}
O_{+}^{+}=\frac{1}{2}\left(\frac{\bar{\omega}}{N}\right)^{1 / 2}\left(Q-\frac{i}{\bar{\omega}} P\right), \\
O_{-}^{+}=\frac{1}{2}\left(\frac{\bar{\omega}}{N}\right)^{1 / 2}\left(Q^{+}-\frac{i}{\bar{\omega}} P^{+}\right),
\end{gathered}
$$

where

$$
\begin{gathered}
Q=\sum_{i=1}^{N}\left(x_{i}+i y_{i}\right) \equiv \sum_{i=1}^{N} q^{i}, \\
P=\sum_{i=1}^{N}\left(p_{x i}+i p_{y i}\right) \equiv \sum_{i=1}^{N} p^{i},
\end{gathered}
$$

and

$$
\bar{\omega}=\left(\omega_{0}^{2}+\frac{\omega_{c}^{2}}{4}\right)^{1 / 2} .
$$

The corresponding frequencies are

$$
\omega_{ \pm}=\bar{\omega} \pm \frac{\omega_{c}}{2}
$$

and it is easy to verify that

$$
\left[L_{z}, O_{ \pm}^{+}\right]= \pm O_{ \pm}^{+} .
$$

The states $O_{ \pm}^{+}|0\rangle$ are normalized to unity and orthogonal, carrying an angular momentum $\left(L_{0} \pm 1\right)$. This exact result stems from the translational invariance of the electronelectron interaction for which $\left[\sum_{i<j}^{N} V_{c}\left(\left|\vec{r}_{i}-\vec{r}_{j}\right|\right), P\right]=0$, and consequently it is independent of the $e-e$ interaction, provided it is local. ${ }^{25}$

It is a simple matter to check that the states $| \pm 1\rangle$ exhaust the $m_{1}$ and $m_{3}$ SR's for the dipole operator $D=\sum_{i=1}^{N} x_{i}$, and that the dipole strength is equally distributed between them. One obtains

$$
\begin{gathered}
m_{1}(D)=\frac{1}{2} N, \\
m_{3}(D)=\frac{1}{2} N\left(\bar{\omega}^{2}+\frac{3}{4} \omega_{c}^{2}\right), \\
|\langle 0|D|+1\rangle|^{2}=|\langle 0|D|-1\rangle|^{2}=\frac{1}{4} \frac{N}{\bar{\omega}},
\end{gathered}
$$

and, for example,

$$
\omega_{+}|\langle 0|D|+1\rangle|^{2}+\omega_{-}|\langle 0|D|-1\rangle|^{2}=\frac{N}{2}=m_{1}(D) .
$$

It is interesting to notice that in the $B=0$ limit, $\omega_{+}=\omega_{-}=$ $E_{3}(D)=\omega_{0}$. This result is independent of the number of electrons in the dot, in agreement with the generalized Kohn theorem. ${ }^{28}$ The parabolicity of the potential is expected to break down when the number of electrons in the dot increases, and the electronic density extends up to the edge of the dot. Departure of $V_{+}(r)$ from the parabolic law originates an $N$ dependence in $\omega_{ \pm}$and $E_{3}(D)$.

The exact solution to Eq. (5) can be used to obtain the static dipole polarizability of the dot $\alpha(D)$, which is twice the $m_{-1}(D)$ sum rule: ${ }^{1,8}$

$$
m_{-1}(D)=\frac{1}{\omega_{+}}|\langle 0|D|+1\rangle|^{2}+\frac{1}{\omega_{-}}|\langle 0|D|-1\rangle|^{2}=\frac{N}{2 \omega_{0}^{2}} .
$$

Consequently, in the parabolic potential approximation the dipole polarizability is independent of the magnetic field. We expect $\alpha(D)$ to be $B$ dependent in the case of a more general confining potential. Using that ${ }^{27}$

$$
\omega_{0}^{2}=\frac{N_{+}}{R^{3}},
$$

we obtain

$$
\alpha(D)=R^{3} \frac{N}{N_{+}} .
$$

The $R$ dependence could have been anticipated from a dimensional analysis. 
Let us finally discuss the nature of the dipole modes with regard to their geometrical shape. If we consider the transition density associated with the excited states $| \pm 1\rangle$

$$
\rho_{t r}(\vec{r})=\left\langle 0\left|\hat{\rho} O_{ \pm}^{+}\right| 0\right\rangle=\left\langle 0\left|\left[\hat{\rho}, O_{ \pm}^{+}\right]\right| 0\right\rangle,
$$

where $\hat{\rho}$ is the $N$-electron density operator

$$
\hat{\rho}=\sum_{i=1}^{N} \delta\left(\vec{r}-\vec{r}_{i}\right)
$$

it is easy to obtain

$$
\rho_{t r}(\vec{r}) \propto \rho^{\prime}(r) e^{ \pm i \theta},
$$

where $\rho(r)$ is the g.s. electronic density, and the prime denotes the $r$ derivative. This transition density is characteristic of an edge excitation.

\section{VARIATIONAL SOLUTION FOR GENERAL AXIALLY SYMMETRIC POTENTIALS}

When the confining potential is no longer parabolic, irrespective of the value of the magnetic field and of the multipolarity of the mode, the equation of motion (5) cannot be solved exactly, and we resort to an approximate method based on Eq. (7). In the dipole case, the natural guess for the $\mathrm{O}_{ \pm}^{+}$operators is

$$
\begin{gathered}
O_{+}^{+}=a_{+}\left(Q-i b_{+} P\right), \\
O_{-}^{+}=a_{-}\left(Q^{+}-i b_{-} P^{+}\right),
\end{gathered}
$$

where $b_{ \pm}$are variational parameters and $a_{ \pm}$have to be determined from the normalization condition. This process can indeed be carried out, and one obtains the dipole spectrum of the system.

At this point, we consider it more convenient to generalize the $Q$ and $P$ operators in such a way that the calculation can be done for any $L$ value. To this end, we have first taken the following $Q_{L}$ :

$$
Q_{L}=\sum_{i=1}^{N} r_{i}^{L} e^{i L \theta_{i}} \equiv \sum_{i=1}^{N} q_{L}^{i} .
$$

This choice is inspired in that $(k r)^{L} e^{i L \theta}$ is the small $k$ expansion of the function $J_{L}(k r) e^{i L \theta}$, which is the restriction to the $z=0$ plane of the general solution of the Laplace equation in cylindrical coordinates. $J_{L}$ is the $L$ Bessel function of the first kind. ${ }^{29}$

Next, we have taken as partner of $Q_{L}$ in the $O^{+}$expression the operator

$$
R_{L}=\sum_{i=1}^{N} q_{L-1} p
$$

where the $i$-particle index is implicit in the particle and momentum coordinates. This choice is again guided by the exact dipole case, since the conmutator $\left[H, Q_{L}\right]$ yields a combination of $Q_{L}$ and $R_{L}$, which reduces to the one we have found in Sec. III when $L=1$.
We have thus considered as $O_{ \pm L}^{+}$operators the following combinations:

$$
\begin{aligned}
& O_{+L}^{+}=a_{+}\left(Q_{L}-i b_{+} L R_{L}\right), \\
& O_{-L}^{+}=a_{-}\left(Q_{L}^{+}-i b_{-} L R_{L}^{+}\right) .
\end{aligned}
$$

Equations (31) reduce to Eqs. (28) for $L=1$, and the proposed operators fulfill Eq. (6). These modes are edge modes whose transition densities read

$$
\rho_{t r}^{ \pm L}(\vec{r})=\left\langle 0\left|\left[\hat{\rho}, O_{ \pm L}^{+}\right]\right| 0\right\rangle \propto r^{L-1} \rho^{\prime}(r) e^{ \pm i L \theta} .
$$

To minimize Eq. (7), it is necessary to have a description of the g.s. $|0\rangle$. We have taken for it the Kohn-Sham sate built from single-particle (s.p.) wave functions obtained in the framework of the CDFT of Ref. 23. We refer the reader to that work for the details. For the present purposes, it is enough to recall that the s.p. wave functions $\phi_{n l \sigma}(r, \theta)$ are separable in $r$ and $\theta$ variables as

$$
\phi_{n l \sigma}(r, \theta)=u_{n l \sigma}(r) e^{-i l \theta},
$$

with $l=0, \pm 1, \pm 2, \ldots$ being the orbital angular momentum of the s.p. state. Upon minimization, one obtains

$$
\omega_{ \pm L}=\frac{1 \pm b_{ \pm}\left(L \omega_{c}+4 L^{2} \widetilde{\omega}_{1}\right)+b_{ \pm}^{2}\left(\widetilde{\omega}_{3}^{2}+L^{3} \omega_{c} \widetilde{\omega}_{1}\right)}{2 b_{ \pm} \pm 2 b_{ \pm}^{2} L^{2} \widetilde{\omega}_{1}},
$$$$
b_{ \pm}=\frac{\sqrt{\widetilde{\omega}_{3}^{2}-3 L^{4} \widetilde{\omega}_{1}^{2}} \pm L^{2} \widetilde{\omega}_{1}}{\widetilde{\omega}_{3}^{2}-4 L^{4} \widetilde{\omega}_{1}^{2}}
$$

$$
a_{ \pm}^{2}=\frac{1}{4 b_{ \pm}\left(m_{1} \pm b_{ \pm} L^{2} \Gamma\right)}
$$

where

$$
\begin{gathered}
\widetilde{\omega}_{3}^{2}=\frac{\tilde{m}_{3}}{m_{1}}+\frac{L}{4} \omega_{c}^{2}, \\
\widetilde{\omega_{1}}=\frac{\Gamma}{m_{1}} \equiv \frac{1}{m_{1}}(L-1) \int d \vec{r} r^{2 L-4} \gamma(r), \\
m_{1}=L^{2} \int d \vec{r} r^{2 L-2} \rho(r), \\
\tilde{m}_{3}=\widetilde{m}_{3}(T)+\widetilde{m}_{3}(e e)+\widetilde{m}_{3}(+e),
\end{gathered}
$$

with

$$
\begin{gathered}
\tilde{m}_{3}(T)=L^{2}(L-1) \int d \vec{r} r^{2 L-4}[L \tau+2(L-2) \lambda], \\
\widetilde{m}_{3}(e e)=4 \pi L^{2} \frac{(2 L-1) ! !}{2^{L} L !} \int_{0}^{\infty} \rho^{\prime}(r) d r\left\{\frac{1}{r} \int_{0}^{r}[2(L+1)\right. \\
\left.\times \rho^{\prime}\left(r^{\prime}\right) r^{\prime 2 L}+\rho^{\prime \prime}\left(r^{\prime}\right) r^{\prime 2 L+1}\right] E_{L}\left(\frac{r^{\prime}}{r}\right) d r^{\prime}
\end{gathered}
$$




$$
\begin{aligned}
& +r^{2 L} \int_{r}^{\infty}\left[\frac{\rho^{\prime}\left(r^{\prime}\right)}{r^{\prime}}-\rho^{\prime \prime}\left(r^{\prime}\right)\right] E_{L}\left(\frac{r}{r^{\prime}}\right) d r^{\prime} \\
& \left.-\frac{2^{L+1} L !}{(2 L+1) ! !} r^{2 L} \rho^{\prime}(r)\right\}-2 \pi L^{2} \int_{0}^{\infty} d r \rho^{\prime}(r) \\
& \times\left\{r ^ { 2 L - 3 } \int _ { 0 } ^ { r } \left[4 r^{\prime 2} \rho^{\prime}\left(r^{\prime}\right)\right.\right. \\
& \left.+r^{\prime 3} \rho^{\prime \prime}\left(r^{\prime}\right)\right] E_{1}\left(\frac{r^{\prime}}{r}\right) d r^{\prime}+r^{2 L} \int_{r}^{\infty} \frac{\rho^{\prime}\left(r^{\prime}\right)}{r^{\prime}} \\
& \left.\left.-\rho^{\prime \prime}\left(r^{\prime}\right)\right] E_{1}\left(\frac{r}{r^{\prime}}\right) d r^{\prime}-\frac{4}{3} r^{2 L} \rho^{\prime}(r)\right\}, \\
\widetilde{m}_{3}(+e)= & -\pi L^{2} \int_{0}^{\infty} d r V_{+}(r)\left[(2 L-1) r^{2 L-2} \rho^{\prime}(r)\right. \\
& \left.+r^{2 L-1} \rho^{\prime \prime}\right] .
\end{aligned}
$$

Besides the particle and kinetic-energy densities

$$
\begin{gathered}
\rho(r)=\sum_{n l \sigma} u_{n l \sigma}^{2}(r), \\
\tau(r)=\left\langle 0\left|\sum_{i} \hat{\nabla} \delta\left(\vec{r}-\vec{r}_{i}\right) \vec{\nabla}\right| 0\right\rangle=\sum_{n l \sigma}\left(u_{n l \sigma}^{\prime 2}+\frac{l^{2}}{r^{2}} u_{n l \sigma}^{2}\right),
\end{gathered}
$$

in these equations we introduced the densities

$$
\begin{gathered}
\gamma(r)=-\sum_{n \ell \sigma} \ell u_{n \ell \sigma}^{2}(r)=r j_{p}(r), \\
\lambda(r)=\sum_{n \ell \sigma} \frac{\ell^{2}}{r^{2}} u_{n \ell \sigma}^{2}(r),
\end{gathered}
$$

as well as the $E_{L}$ function

$$
E_{L}(z) \equiv \frac{\pi}{2} F\left(-\frac{1}{2}, L+\frac{1}{2} ; L+1 ; z^{2}\right),
$$

where $F$ is the hypergeometrical function. ${ }^{29}$ The density $\lambda(r)$ represents a sort of centrifugal kinetic-energy density. It can be shown that in the zeroth-order Thomas-Fermi (TF) approximation $\lambda(r)=\frac{1}{2} \tau(r)$, similar to the $\lambda(r)=\frac{2}{3} \tau(r)$ relation holding in three dimensions. ${ }^{30}$ The function $j_{p}(r)$ is the paramagnetic current density. ${ }^{23}$ It is worthwhile to see that, at $B=0, \gamma(r)$ vanishes due to time-reversal invariance. Its contribution at high magnetic fields is crucial to have wellbehaved $B$-dependent collective energies. Equations (34)(42) constitute the main outcome of the present section, and will be used in Sec. VI to obtain numerical results within CDFT.

The goal of describing the multipole modes in a situation as general as possible makes these expressions look very cumbersome at first sight. However, the following can be noted.

(i) For $L=1$ and the parabolic $\mathrm{V}_{+}$, one recovers the exact solution. (ii) For any $L$ and zero magnetic field, $b_{ \pm}=1 / \widetilde{\omega}_{3}$ and $O_{ \pm L}^{+}|0\rangle$ merge into a degenerate state of energy

$$
\omega_{ \pm L}=\left(\frac{\tilde{m}_{3}}{m_{1}}\right)^{12} .
$$

This is precisely the $B=0$ scaling energy $E_{3}\left(Q_{L}\right)$, since $m_{1}$ is actually the general $m_{1}\left(Q_{L}\right)$ sum rule, and, as is outlined in the Appendix, $\widetilde{m}_{3}$ reduces to the zero magnetic field $m_{3}\left(Q_{L}\right)$ sum rule making $\gamma(r)=0$. This result is the variational analog of the case we have discussed for the dipole mode in a parabolic $V_{+}$.

(iii) In the $L=1$ case, irrespective of $B$, all terms in $\tilde{m}_{3}$ but $\tilde{m}_{3}(+e)$ vanish, and for any axially symmetric ionic potential $V_{+}(r)$ we find

$$
\omega_{ \pm 1}=\left(\frac{\omega_{c}^{2}}{4}+\frac{1}{2 N} \int d \vec{r} \Delta V_{+}(r) \rho(r)\right)^{1 / 2} \pm \frac{\omega_{c}}{2}
$$

which reduces again to the exact case if $\mathrm{V}_{+}=\frac{1}{2} \omega_{0}^{2} \mathrm{r}^{2}$.

(iv) For any $B$, the states $| \pm L\rangle$ and their energies satisfy the $m_{1}$ sum rule, i.e.,

$$
\begin{aligned}
m_{1}\left(Q_{L}\right)= & L^{2} \int d \vec{r} r^{2 L-2} \rho(r)=\omega_{+}\left|\left\langle 0\left|\left[Q_{L}, O_{+L}^{+}\right]\right| 0\right\rangle\right|^{2} \\
& +\omega_{-}\left|\left\langle 0\left|\left[Q_{L}, O_{-L}^{+}\right]\right| 0\right\rangle\right|^{2} .
\end{aligned}
$$

This implies that, although $O_{ \pm L}^{+}|0\rangle$ are only approximate solutions to the $L$-polar excitation spectrum, within our method there are no other excited collective states corresponding to that multipolarity built as coherent superposition of one electron-hole states. The sum rule

$$
\begin{aligned}
m_{3}\left(Q_{L}\right)= & \tilde{m}_{3}(T)+\frac{3}{4} L^{2} \omega_{c}^{2} m_{1}+3 \omega_{c} L^{3} \Gamma+\tilde{m}_{3}(e e) \\
& +\tilde{m}_{3}(+e)
\end{aligned}
$$

is also fulfilled. Moreover, the square transition amplitudes to the $| \pm L\rangle$ states are equal,

$$
\left|\left\langle 0\left|\left[Q_{L}, O_{+L}^{+}\right]\right| 0\right\rangle\right|^{2}=\left|\left\langle 0\left|\left[Q_{L}, O_{-L}^{+}\right]\right| 0\right\rangle\right|^{2} .
$$

The fulfillment of $m_{1}$ and $m_{3}$ constitutes a rather stringent test on our variational method.

(v) It is worth noticing that in all cases, there is no explicit contribution to these formulas arising from the exchange correlation energy terms in the CDFT Hamiltonian. This is because $Q_{L}$ is a solution of the two-dimensional Laplace equation. A similar result holds in three dimensions within the LDA if one takes for $Q_{L}$ a solution of the corresponding Laplace equation. ${ }^{31}$

\section{EDGE WAVES IN NEUTRAL LARGE DOTS AT $B=0$}

It is possible to use the scaling energy $E_{3}$, which is a good approximation of the collective excitation energy at $B=0$, to obtain the dispersion relation of the edge waves in the case of a neutral large dot. Under these conditions, the g.s. electronic density is constant everywhere apart from a narrow region along the border of the disk. Let $\rho_{0}=N /\left(\pi R^{2}\right)$ be that 
density, and let $a$ be the width of the edge region. Using the zeroth-order TF approximation to the kinetic and centrifugal kinetic-energy densities

$$
\begin{gathered}
\tau=\pi \rho^{2}, \\
\lambda=\frac{1}{2} \tau,
\end{gathered}
$$

one obtains, from Eqs. (38) and (40)-(42),

$$
\begin{gathered}
m_{1}=\pi \rho_{0} L R^{2 L}, \\
\tilde{m}_{3}(T)=2 L^{2}(L-1) \pi^{2} \rho_{0}^{2} R^{2 L-2}, \\
\tilde{m}_{3}(e e)+\tilde{m}_{3}(+e)=4 \pi L^{2} R^{2 L-1} \rho_{0}^{2} \frac{(2 L-1) ! !}{2^{L} L !} F_{L}(z),
\end{gathered}
$$

with $z=1-O(a / R)$ at least, and

$$
F_{L}(z) \equiv \frac{\pi}{2} F\left(\frac{1}{2}, L+\frac{1}{2} ; L+1 ; z^{2}\right)
$$

which diverges at $z=1$. Physically, this divergency is associated with the known divergency of the electric field at the edge of the disk in the case of a constant electron density; ${ }^{32}$ see below.

Thus

$$
E_{3}^{2}=2 \pi \rho_{0} \frac{L(L-1)}{R^{2}}+4 \frac{\rho_{0}}{R} \frac{(2 L-1) ! !}{2^{L}(L-1) !} F_{L}(z)
$$

$F_{L}(z)$ can be written as a function of $F_{0}(z)$, which is the elliptic function $\mathbf{K}(z){ }^{29}$

$$
\frac{(2 L-1) ! !}{2^{L} L !} F_{L}(z)=F_{0}(z)-\sum_{m=1}^{L} \frac{1}{2 m-1} .
$$

Using this, we obtain

$$
E_{3}^{2}=2 \pi \rho_{0} \frac{L(L-1)}{R^{2}}+4 \rho_{0} \frac{L}{R}\left[\frac{1}{2} F_{1}(z)+1-\sum_{m=1}^{L} \frac{1}{2 m-1}\right] .
$$

On the other hand, $F_{1}(z)$ can be related to the electric field $E(r)$ generated by the electrons at the edge of the disk:

$$
2 \rho_{0} F_{1}(z)=-\left.\frac{d V}{d r}\right|_{r \rightarrow R}=E(r)_{r \rightarrow R} .
$$

The divergency of $E(r)$ at $r=R$ is removed when one considers the existence of the edge width: ${ }^{32}$

$$
E(R)=2 \rho_{0} \ln \left(\gamma \frac{R}{a}\right)
$$

where $\gamma$ is a constant that depends on the precise way the electronic density goes to zero at $R$. Thus

$$
\begin{aligned}
E_{3}^{2}= & 2 \pi \rho_{0} \frac{L(L-1)}{R^{2}}+4 \rho_{0} \frac{L}{R}\left[\frac{1}{2} \ln \left(\gamma \frac{R}{a}\right)+1\right. \\
& \left.-\sum_{m=1}^{L} \frac{1}{2 m-1}\right] .
\end{aligned}
$$

If $L \gg 1$ but still $N \gg L$, that expression can be further elaborated since

$$
\sum_{m=1}^{L} \frac{1}{2 m-1} \sim \frac{1}{2}(C+\ln L)+\ln 2,
$$

where $C$ is the Euler constant $0.5772 \ldots$ Introducing the wave vector $q=L / R$, and keeping only the leading $q$ term, we obtain

$$
E_{3}=\omega(q)=\sqrt{2 \rho_{0} q \ln \left(q_{0} / q\right)},
$$

where $q_{0}=\gamma /(a \beta)$ and $\beta=0.964$.

Result (65) can also be obtained using classical hydrodynamics. ${ }^{32}$ It is worthwhile to notice that the above$\omega(q)$ model differs from that obtained within the edgemagnetoplasmon model (Refs. 15-17):

$$
\omega(q)=\left(\frac{4 \pi}{3} \rho_{0} q\right)^{1 / 2}
$$

which is $\sqrt{2 / 3}$ times that of the two-dimensional plasma frequency. We would like to stress that, to obtain the $\sqrt{q \ln \left(q_{0} / q\right)}$ dispersion relation, it is crucial to take into account width effects in the electronic density. These effects are important in quantum dots, where the number of electrons usually is much less than the number of ions, and in any case the electron density has a non-negligible edge region.

\section{NUMERICAL RESULTS}

We have applied the method of Sec. IV to dots made of $N=6,20,30,42$, and 56 electrons. We have taken the values of $g^{*}, \epsilon$ and $m^{*}$ indicated after Eq. (1), and $N_{+}=125$, i.e., a positive density of $\sim 4 \times 10^{11}$ ions $/ \mathrm{cm}^{2}$. This corresponds to a GaAs disk of about $1000-\AA$ radius modeling the positive background.

The g.s. of the dots has been obtained using the CDFT of Ref. 23. We checked that we reproduce their results when we use the same $V_{+}$. For large dots and intense magnetic fields, sometimes one has to face severe convergence problems in the solution of the Kohn-Sham equations. Rather than a deficiency of the numerical algorithm, we consider it as a consequence of an inherent characteristic of the system under study, namely the existence of a very dense s.p. energy spectrum. To overcome it, on the one hand, and to carry out the calculations under conditions closer to the experimental ones, on the other hand, we have found it convenient to approach the description of the collective spectrum as the low-temperature limit of the results obtained from the finite temperature generalization of the formalism of Sec. II (see Ref. 33), and of the Kohn-Sham equations (see, for example, Ref. 34). Consequently, the numerical results we discuss below have been obtained at a temperature $T \sim 1-2 \mathrm{~K}$. A comparison with several cases in which the $T=0$ calculation is 


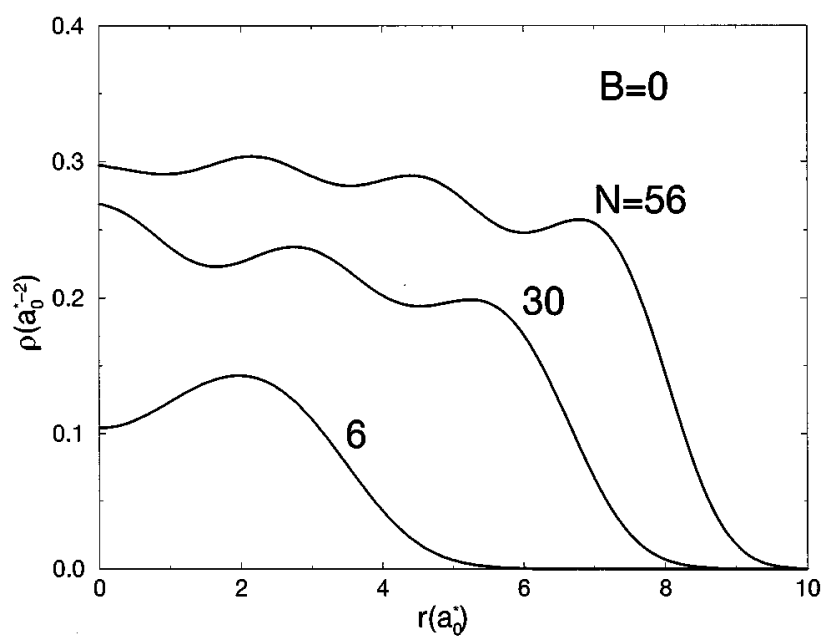

FIG. 1. Electronic densities in $\left(a_{0}^{*}\right)^{-2}$ as a function of $r$ in $a_{0}^{*}$, for dots with $N=6,30$, and 56 electrons, and $B=0$.

easy to converge, allows us to state that the small temperature we use does not influence the results here presented. Thermal effects on the collective spectrum of quantum dots will be described in detail elsewhere.

Figures 1 and 2 show the electronic densities corresponding to dots with $N=6,30$, and 56 electrons for $B=0$, and for $B=5 \mathrm{~T}$, respectively. Figures $3-5$ display the $B$-dependent, multipolar spectrum of the same dots up to $L=4$. This is the interesting region where the crossing between $\omega_{+L}$ and $\omega_{-L^{\prime}}$ branches may occur and has been experimentally observed. The energies are drawn in meV, and the magnetic field in $\mathrm{T}$. These figures show that as the $\omega_{-L}$ energies go to zero, they may reach a value comparable to the electron-hole s.p. energy difference at a rather moderate $B$ value. When this happens, the collective state lies within the s.p. excitations region, and experiences a strong Landau damping, losing its collectivity and eventually being washed out. This is one of the reasons why the $\omega_{+L}$ branch has been experimentally observed up to higher $B$ values than the $\omega_{-L}$ branch. $^{7}$

The structures appearing along the $L>1$ curves are due to drastic changes in the particle, kinetic and paramagnetic current densities of the dot arising from the effect of $B$ through

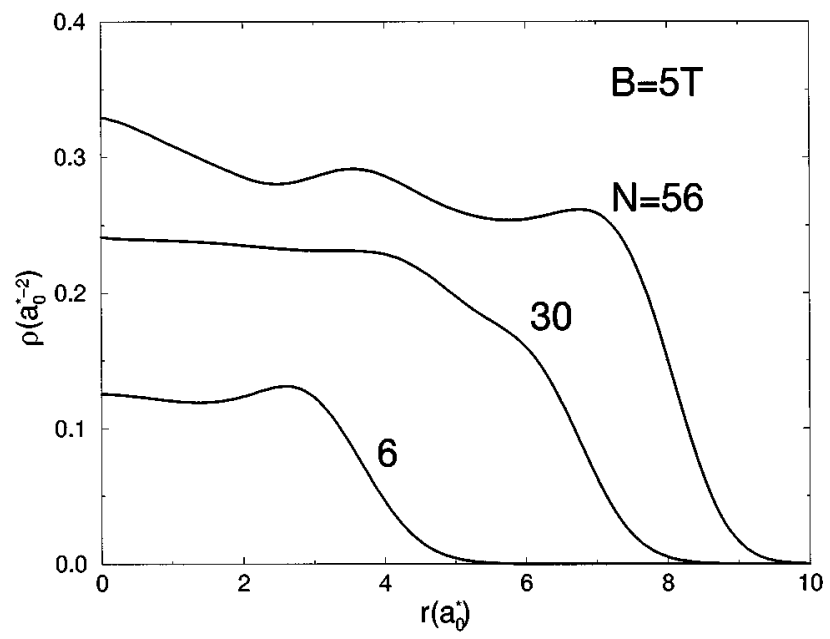

FIG. 2. Same as Fig. 1, for $B=5 \mathrm{~T}$.

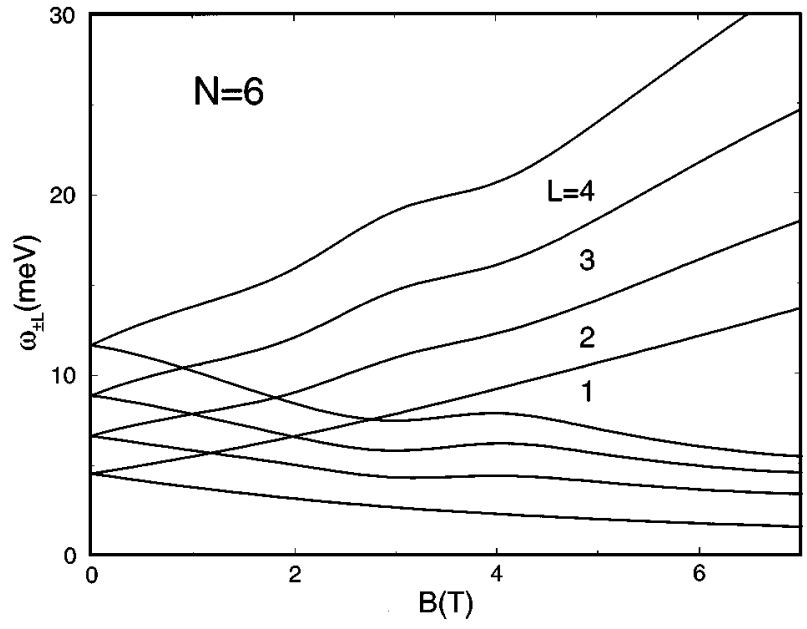

FIG. 3. $\omega_{ \pm L}$ energies in $\mathrm{meV}$ as a function of $B$ in $\mathrm{T}$ for $L=1-4$, corresponding to $N=6$.

the exchage-correlation energy, which has a profound influence on them (see Fig. 3 of Ref. 23). The structures in the $\omega_{ \pm L}$ branches roughly correspond to values of $B$ at which the total g.s. spin has a minimum. For example, for $N=6$ we have that $2 S$ is equal to 2 at $B=1 \mathrm{~T}$, to zero at $B=2 \mathrm{~T}$, and to 2 at $B \sim 3 \mathrm{~T}$. For $N=56$, one has that $2 S=13$ at $B=4 \mathrm{~T}$, 7 at $B \sim 5 \mathrm{~T}$, and 8 at $B=6 \mathrm{~T}$. For the $N=6 \mathrm{dot}$, the rising of the $\omega_{+L>1}$ curves at $B \sim 5 \mathrm{~T}$ is due to the full alignment of the electron spins. No similar risings show up for $N=30$ and 56 because for them, the alignment occurs at $B$ values higher than those displayed in the figures.

It can be seen from Figs. 3-5 that the crossing between $\omega_{+1}$ and $\omega_{-L}$ branches does not follow a clear $N$ systematics. We have also plotted in Fig. 4 the $E_{3}\left(Q_{L}\right)$ energies for $L=1$ and 4 (dashed lines). The scaling energy is reproducing $\omega_{+L}$ to within $10-20 \%$, since the $\omega_{-L}$ branch is contributing to $m_{3}\left(\mathrm{Q}_{L}\right)$ with the same weight as the $\omega_{+L}$ one, and the negative $B$-dispersion energies are going to zero rather slowly.

We also display in Fig. 5 (dashed lines), the collective energies obtained for a parabolic potential whose $\omega_{0}$ has been fixed to $5.6 \mathrm{meV}$ in order to reproduce the $\omega_{1}$ energy at

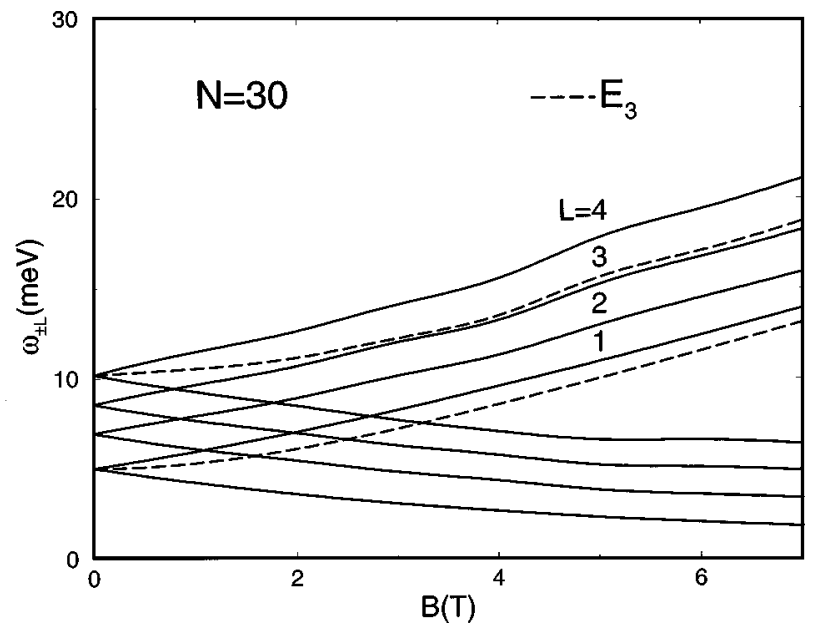

FIG. 4. Same as Fig. 3 for $N=30$. The dashed lines are the $E_{3}\left(Q_{L}\right)$ energies for $L=1$ and 4 . 


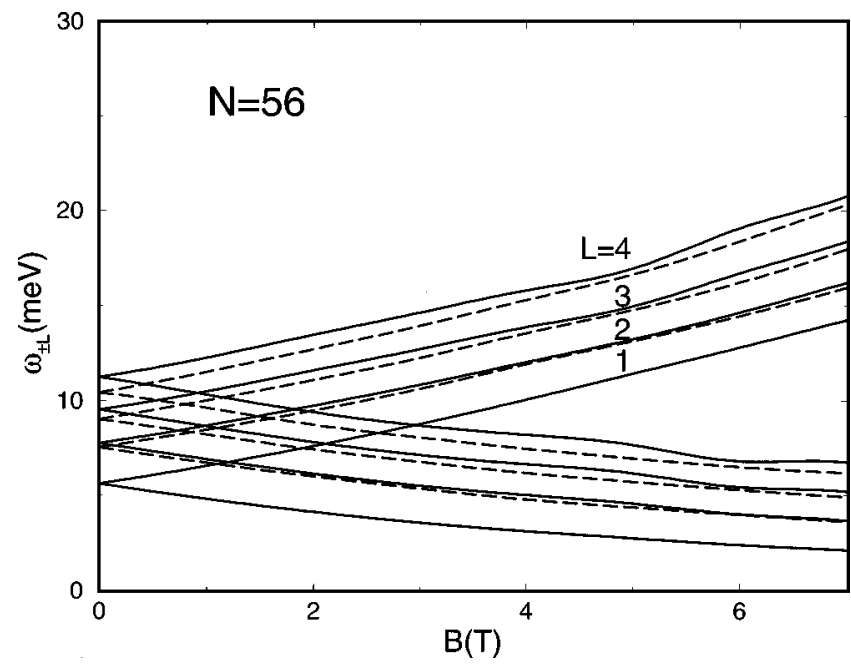

FIG. 5. Same as Fig. 3 for $N=56$. The dashed lines represent $\omega_{ \pm L>1}$ obtained using a parabolic potential instead of the one generated by the disk.

$B=0$. It is worth noticing that this value does not equal the one which fits the Coulomb potential generated by the 1000$\AA$-radius disk charged with $N_{+}=125$ ions near the origin, which is $4.4 \mathrm{meV}$, and consequently, $\omega_{0}$ has to be interpreted here as an effective parameter to reproduce the dipole energy at zero magnetic field. For $L=1$, to the scale of the figure both calculations coincide. It may be seen that the $B$ slopes of the $\omega_{ \pm L}$ branches for $L=2-4$ are roughly the same for the parabolic and disk confining potentials.

At $B=0$, Figs. 6 and 7 show, for $N=6$ and 56, respectively, the different relative contributions to $m_{3}$ coming from kinetic and Coulomb energies, as a function of $L$. The Coulomb energy is also decomposed into $e-e$ and dot-electron $(+e)$ energies. These figures show that, for small $L$ values, $m_{3}$ is dominated by the $(+e)$ contribution, the kinetic and $(e-e)$ ones being of minor importance. However, for a fixed $N$ the kinetic contribution eventually takes over the Coulomb energy contribution. This occurs at an angular momentum

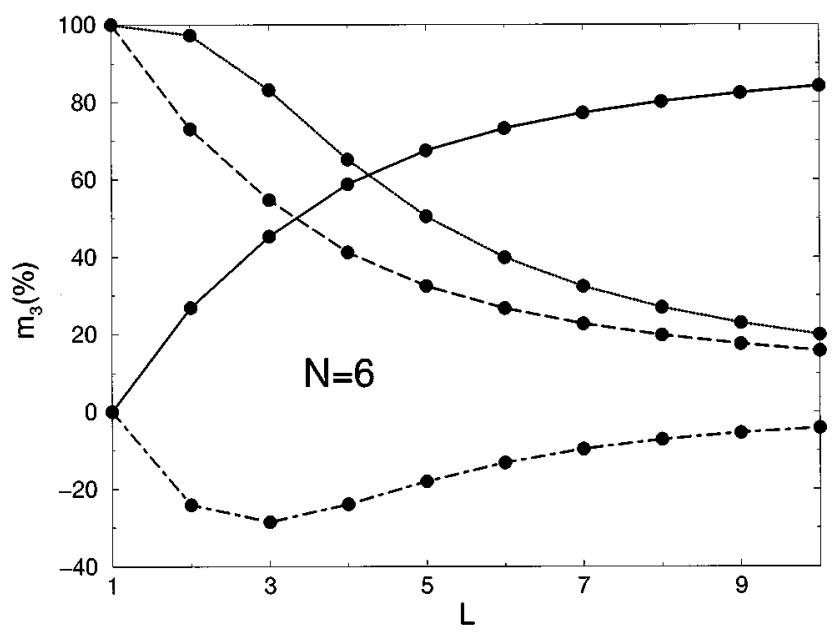

FIG. 6. Decomposition of $m_{3}\left(Q_{L}\right)$ into kinetic (solid line), total Coulomb (dashed line), ( $e-e)$-component (dash-dotted line), and $(+e)$-component (dotted line) contributions as a function of $L$ for the $N=6$ dot at $B=0$. The lines are drawn to guide the eye.

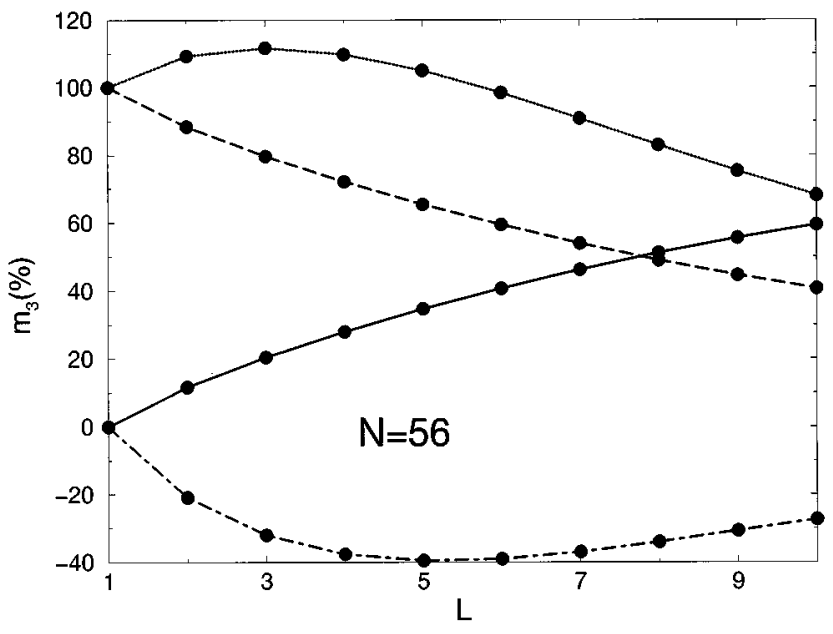

FIG. 7. Same as Fig. 6 for $N=56$.

$L_{\text {cr }}$ which increases when $N$ increases. Since $Q_{L}$ behaves as $\sim r^{L}$, the higher the $L$, the more external its influence on the electronic density. This means that for large enough $L$, it just acts on the s.p. wave functions having the larger $l$ angular momentum and radial quantum number $n$. Consequently, it no longer generates collective but s.p. excitations. As collectivity has its origin in interparticle effects, it was argued in Ref. 14 that $L_{\mathrm{cr}}$ roughly represents the largest $L$ collective mode the system can sustain, since for $L>L_{\mathrm{cr}}$ the restoring force represented by $m_{3}$ is basically determined by an independent particle property like the kinetic energy. This criterion yields $L_{\mathrm{cr}} \sim 4,6$, and 8 for $N=6,30$, and 56, respectively.

In Fig. 8 we represent the energy of modes with $L=1-4$ at $B=0$, as a function of the number of electrons in the dot. The ratios $\omega_{3} / \omega_{1}$ and $\omega_{2} / \omega_{1}$ have, for $N>20$, average values 1.68 and 1.37, respectively, instead of 3 and 2 as corresponds to the harmonic-oscillator sequence. It is apparent from that figure the $N$ dependence of $\omega_{L}$. As we already discussed, only for the dipole mode in the case of a parabolic confining potential does one have $\omega_{1}=\omega_{0}$. The deviation

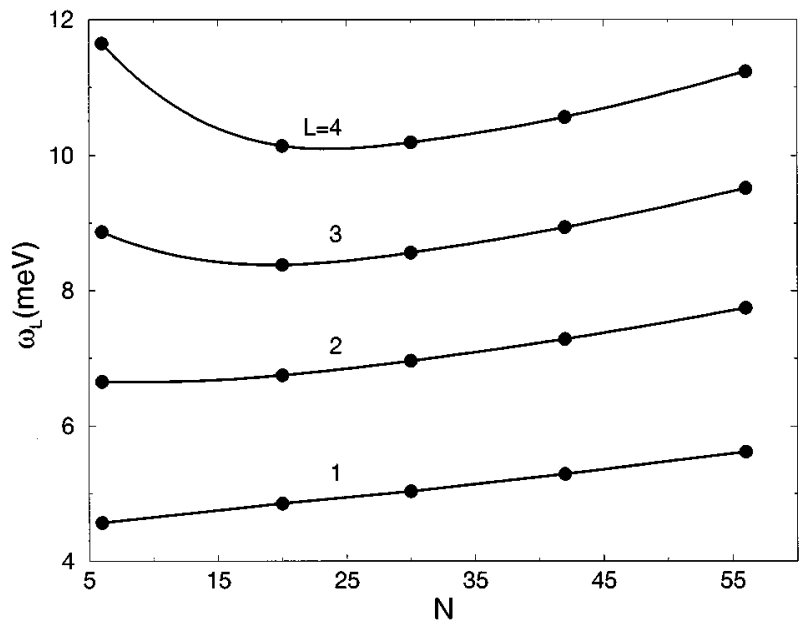

FIG. 8. Zero magnetic field $\omega_{L}$ energies in $\mathrm{meV}$ for $L=1-4$, as a function of $N$. 


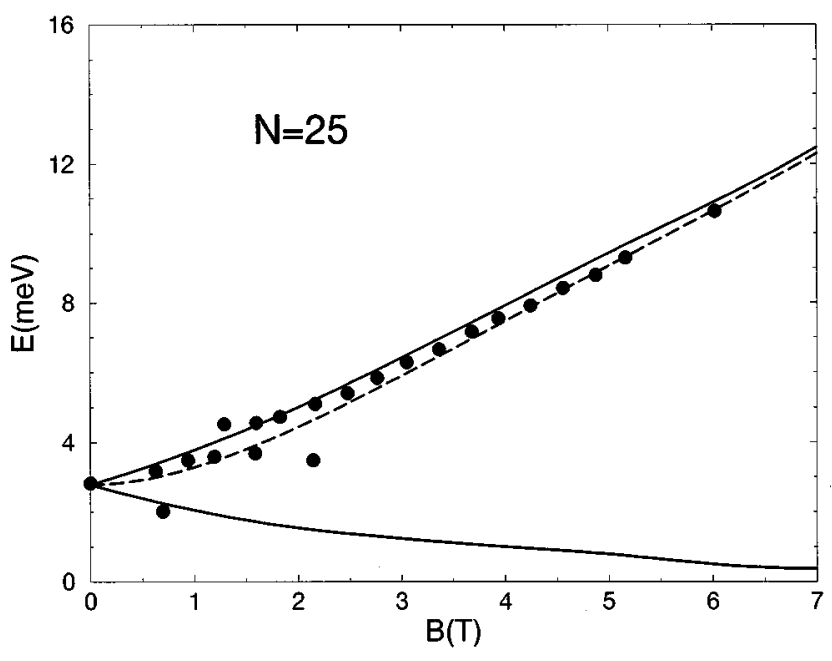

FIG. 9. $\omega_{ \pm 1}$ (solid lines) and $E_{3}\left(Q_{1}\right)$ (dashed line) energies in $\mathrm{meV}$ as a function of $B$ in T, for a dot of $R=1000 \AA, N_{+}=28$, and $N=25$. The values of $\epsilon, g^{*}$, and $m^{*}$ are those of GaAs given in the text. The points are experimental results taken from Ref. 6.

from that rule becomes more and more important as the number of electrons in the dot increases. This was already discussed in Ref. 19. The $N$ dependence of $\omega_{L>1}$ is more complex, and this is due to the interplay between kinetic and Coulomb energy contributions to the excitation energy. It can be easily understood from Eq. (58), which shows that the contribution of the kinetic energy term is important for small $N$ and increases with increasing $L$. For fixed $L$, the Coulomb energy eventually dominates, and $\omega_{L>1}$ depends on $N$ in a way similar to the dipole mode.

Finally, we have also studied the collective spectrum of the $N=6$ dot in the parabolic potential with $\omega_{0}=5.6 \mathrm{meV}$, and have found that, as expected, the $\omega_{L>1}$ energies at $B=0$ depend on $N$. For example, $\omega_{2}$ decreases from 8.2 to 7.5 $\mathrm{meV}, \omega_{3}$ decreases from 10.9 to $9.0 \mathrm{meV}$, and $\omega_{4}$ decreases from 14.3 to $10.4 \mathrm{meV}$ when one goes from $N=6$ to 56 .

So far, we presented a systematic study carried out under well-defined conditions which could render a comparison with the results of a given experiment difficult, since there are several variables that have to be fixed at the experimental values to permit a sensible comparison. To end this section, we compare some results obtained within our formalism with the experimental data of Ref. 7. This is possible only in part because, in that work, the $L=1$ and 2 well-defined branch were obtained for a dot made of a large number of electrons, $N=210$. At present, this is too large a value for us to deal with microscopically. Let us remind the reader that the s.p. wave functions behave near the origin as $r^{|l|}$ (see, for example, Ref. 24), and since the s.p. levels are nondegenerate when $B \neq 0$, large $N$ 's imply large s.p. angular momenta. Consequently, we have only attempted to describe the $N=25, R=1000-\AA$ dot. This can be inferred from the value of the dipole energy Demel et al. found at $B=0$, that $N_{+} \sim$ 28 . For this dot, the ratio $\omega_{2} / \omega_{1}$ they obtained is around 2 , a value we are unable to reproduce, whereas for the $N=210$ dot it is $\sim 1.5$, in better agreement with our systematics. Nevertheless, it can be seen from Fig. 9 that $\omega_{+1}$ and $E_{3}\left(Q_{1}\right)$ nicely reproduce the positive $B$-dispersion branch, which is the only one for which a detailed comparison is possible.

We notice that the influence of the dot-dot interaction has not been considered in the comparison with the experiment. That effect was estimated in Ref. 25, and it can be shown to have a negligible influence on the frequency of the collective modes for the experimental dot array of Ref. 7 because the dot-dot distance is much larger (about ten times larger) than the dot radius.

\section{SUMMARY}

In this work we used a variational approach similar to the one proposed by Feynmann in the case of liquid ${ }^{4} \mathrm{He}$, to describe the multipole spectrum of quantum dots. One of the merits of the method is that quantum and finite-size effects can be taken into account. It may be easily applied to dots hosting several tens of electrons. Rather than in the method itself, this limitation has its origin in technical difficulties inherent to current microscopic approaches to handle a large number of electrons in intense magnetic fields.

The ground state of the dots has been described within current density functional theory. This has permitted us to incorporate, in a description of the spectrum, an exchangecorrelation energy contribution that takes naturally into account current effects appearing at nonzero magnetic fields.

We presented a systematic description of edge modes up to $L=4$ in the region of interest to describe level crossing at nonzero magnetic fields. We gave explicit formulas for the $m_{1}$ and $m_{3}$ sum rules corresponding to the general multipole operator Eq. (29), which reduce to very simple expressions in the dipole case, Eqs. (A23). These sum rules are exact within CDFT, and may be of interest to check the accuracy of any detailed calculation of the $L$-mode strength, similarly to the way they are currently used within time-dependent local-density approximation. ${ }^{35}$ Besides this practical application, it is worth noticing that there are few studies in the literature of an $m_{3}$ sum rule corresponding to a physical situation where time-reversal invariance is violated (see, for example, Refs. 36 and 37 for the three-dimensional polarized electron gas).

For large neutral dots at zero magnetic field, we showed that the classical hydrodynamic dispersion law for edge waves $\omega(q) \sim \sqrt{q \ln \left(q_{0} / q\right)}$ holds when quantum and finitesize effects are taken into account. Finally, we also showed that in the case of a parabolic potential, the dipole mode can be exactly solved, yielding the well-known classical formula for $\omega_{ \pm 1}$. The exactness of the dipole collective spectrum was stressed in Refs. 24 and 25. Here we obtained it in a different way, and went a step further explaining the structure of the $| \pm 1\rangle$ collective states. This allowed us to obtain a variational solution valid for any axially symmetric lateral confining potential.

\section{ACKNOWLEDGMENTS}

It is a pleasure to thank Luis Brey for a useful correspondence. This work was performed under Grant Nos. PB951249 and PB95-0492 from CICYT, SAB95-0388 from DGID, Spain, and GRQ94-1022 from Generalitat of Catalunya. 


\section{APPENDIX}

In this appendix we give some hints about how to derive Eqs. (34)-(42), and the sum rules $m_{1}$ and $m_{3}$ corresponding to the external one-body operator $Q_{L}$ of Eq. (29), which are given in Eqs. (38) and (51). The details of the method we use here can be found in Refs. 1,8,14 and 31 .

We fix our attention on the operator $O_{+L}^{+}$of Eq. (31); the operator $O_{-L}^{+}$is handled similarly. The double conmutator in the numerator of Eq. (7) can be decomposed into three pieces:

$$
\begin{aligned}
{\left[O_{+L},\left[H, O_{+L}^{+}\right]\right]=} & a_{+}^{2}\left\{\left[Q_{L}^{+},\left[H, Q_{L}\right]\right]\right. \\
& +i b_{+} L\left(\left[R_{L}^{+},\left[H, Q_{L}\right]\right]-\left[Q_{L}^{+},\left[H, R_{L}\right]\right]\right) \\
& \left.+b_{+}^{2} L^{2}\left[R_{L}^{+},\left[H, R_{L}\right]\right]\right\} .
\end{aligned}
$$

Splitting the Hamiltonian Eq. (3) into a one-body term $H_{0}$ and a two-body term $V=\sum_{i<j} V\left(\left|\vec{r}_{i}-\vec{r}_{j}\right|\right)$, and using that $Q_{L}$ is a local operator which commutates with $V$, one obtains

$$
\left[H, Q_{L}\right]=\left[H_{0}, Q_{L}\right]=-i L R_{L}+\frac{1}{2} L \omega_{c} Q_{L},
$$

where we used the conmutation relations

$$
\left[T, Q_{L}\right]=-i L R_{L}, \quad\left[L_{z}, Q_{L}\right]=L Q_{L} .
$$

From Eq. (A2) and

$$
\left[Q_{L}^{+}, P\right]=2 i L Q_{L-1}^{+}, \quad\left[L_{z}, P\right]=P,
$$

it is then inmediate to derive, for the first two terms of Eq. (A1), the results

$$
\begin{gathered}
\left\langle 0\left|\left[Q_{L}^{+},\left[H, Q_{L}\right]\right]\right| 0\right\rangle=2 L^{2} \int r^{2 L-2} \rho(r) d \vec{r}=2 m_{1}, \\
\left\langle 0\left|\left[R_{L}^{+},\left[H, Q_{L}\right]\right]-\left[Q_{L}^{+},\left[H, R_{L}\right]\right]\right| 0\right\rangle=-2 i\left(\omega_{c} m_{1}+4 L \Gamma\right),
\end{gathered}
$$

where

$$
\Gamma=\frac{1}{4}\left\langle 0\left|\left[R_{L}^{+}, R_{L}\right]\right| 0\right\rangle=(L-1) \int d \vec{r} r^{2 L-4} \gamma(r) .
$$

To evaluate $\left[R_{L}^{+},\left[H, R_{L}\right]\right]$, we first calculate

$$
\left[H, R_{L}\right]=\left[H_{0}, R_{L}\right]+\left[V, R_{L}\right] .
$$

The first term gives

$$
\begin{aligned}
{\left[H_{0}, R_{L}\right]=} & -i(L-1) \sum_{i=1}^{N} q_{L-2} p^{2}+\frac{1}{2} L \omega_{c} R_{L}+\frac{i}{4} \omega_{c}^{2} Q_{L} \\
& +i \sum_{i=1}^{N}\left(\partial_{x}+i \partial_{y}\right) V_{+}\left(r_{i}\right) q_{L-1}
\end{aligned}
$$

and it is then straightforward to recover the results of Eqs. (40) and (42) for

$$
\widetilde{m}_{3}(T)=\frac{L^{2}}{2}\left\langle 0\left|\left[R_{L}^{+},-i(L-1) \sum_{i=1}^{N} q_{L-2} p^{2}\right]\right| 0\right\rangle
$$

and

$$
\tilde{m}_{3}(+e)=\frac{L^{2}}{2}\left\langle 0\left|\left[R_{L}^{+}, i \sum_{i=1}^{N}\left(\partial_{x}+i \partial_{y}\right) V_{+}\left(r_{i}\right) q_{L-1}\right]\right| 0\right\rangle
$$

The remaining two terms in Eq. (A9) give

$$
\left\langle 0\left|\left[R_{L}^{+}, \frac{L}{2} \omega_{c} R_{L}\right]\right| 0\right\rangle=2 L \omega_{c} \Gamma,
$$

which yields the Eq. (37) term, and

$$
\left\langle 0\left|\left[R_{L}^{+}, \frac{i}{4} \omega_{c}^{2} Q_{L}\right]\right| 0\right\rangle=\frac{1}{2} \frac{\omega_{c}^{2}}{L} m_{1}
$$

which yields the second term in Eq. (36).

There remains the problem of evaluating the $\left\langle 0\left|\left[R_{L}^{+},\left[V, R_{L}\right]\right]\right| 0\right\rangle$ term. We have done this within CDFT by scaling the CDFT g.s. as

$$
|\eta\rangle=e^{\eta R_{L}}|0\rangle
$$

and then calculating the derivative

$$
\left.\frac{1}{2} \frac{\partial^{2}}{\partial \eta^{2}}\langle\eta|V| \eta\rangle\right|_{\eta=0}
$$

where

$$
\langle\eta|V| \eta\rangle=\frac{1}{2} \int \frac{\rho_{\eta}\left(\vec{r}_{1}\right) \rho_{\eta}\left(\vec{r}_{2}\right)}{\left|\vec{r}_{1}-\vec{r}_{2}\right|} d \vec{r}_{1} d \vec{r}_{2}
$$

and

$$
\rho_{\eta}(\vec{r})=\left\langle\eta\left|\sum_{i=1}^{N} \delta\left(\vec{r}-\vec{r}_{i}\right)\right| \eta\right\rangle=\rho+\eta \rho_{1}+\eta^{2} \rho_{2}+\cdots,
$$

with

$$
\rho_{1}=-L r^{L-1} \rho^{\prime}(r) e^{i L \theta},
$$

$$
\rho_{2}=\frac{1}{2} L^{2}(2 L-1) r^{2 L-3} \rho^{\prime}(r)+\frac{1}{2} L^{2} r^{2 L-2} \rho^{\prime \prime}
$$

From Eqs. (A14)-(A19), one obtains the result Eq. (41):

$$
\tilde{m}_{3}(e e)=\frac{L^{2}}{2}\left\langle 0\left|\left[R_{L}^{+},\left[V, R_{L}\right]\right]\right| 0\right\rangle .
$$

It is seen that the exchange-correlation energy does not give any explicit contribution to $\widetilde{m}_{3}(e e)$. However, it affects g.s. magnitudes like $\rho(r)$ and the other densities, thus implicitly influencing all these quantities.

The commutator $\left[\mathrm{O}_{+L}^{+}, \mathrm{O}_{+L}\right]$ of the denominator of Eq. (7) is easily evaluated to be 


$$
\begin{aligned}
\left\langle 0\left|\left[O_{+L}^{+}, O_{+L}\right]\right| 0\right\rangle= & a_{+}^{2}\langle 0|\left[Q_{L}-i b_{+} L R_{L}, Q_{L}^{+}\right. \\
& \left.+i b_{+} L R_{L}^{+}\right]|0\rangle \\
= & 4 a_{+}^{2}\left(b_{+} m_{1}+b_{+}^{2} L^{2} \Gamma\right) .
\end{aligned}
$$

The cubic energy weighted sum rule Eq. (10) for the external operator $Q_{L}$ is given by

$$
m_{3}=\frac{1}{2}\left\langle 0\left|\left[\left[H,-i L R_{L}^{+}+\frac{1}{2} L \omega_{c} Q_{L}^{+}\right],-i L R_{L}+\frac{1}{2} L \omega_{c} Q_{L}\right]\right| 0\right\rangle,
$$

where we employed Eq. (A2). Using the previous result it is easy to recover the expression Eq. (51), which at $B=0$ reduces to $\widetilde{m}_{3}$ [Eq. (39)] with $\gamma(r)=0$.
Let us finally indicate that, for the dipole operator, the $m_{1}$ and $m_{3}$ sum rules for any value of $B$ and an axially symmetric confining potential $V_{+}$have the simple expressions

$$
m_{1}(D)=\frac{N}{2}
$$

$$
m_{3}(D)=\frac{N}{2} \omega_{c}^{2}+\frac{1}{4} \int \Delta V_{+}(r) \rho(r) d \vec{r} .
$$

*Permanent address: Dipartimento di Fisica, Università di Trento, 38050 Povo, Italy.

${ }^{1}$ E. Lipparini and S. Stringari, Phys. Rep. 175, 103 (1989).

${ }^{2}$ W. Ekardt, Phys. Rev. Lett. 52, 1925 (1984).

${ }^{3}$ M. Brack, Rev. Mod. Phys. 65, 677 (1993).

${ }^{4}$ W. de Heer, Rev. Mod. Phys. 65, 611 (1993).

${ }^{5}$ M. Rocca, Surf. Sci. Rep. 22, 1 (1995).

${ }^{6}$ Ch. Sikorski and U. Merkt, Phys. Rev. Lett. 62, 2164 (1989).

${ }^{7}$ T. Demel, D. Heitmann, P. Grambow, and K. Ploog, Phys. Rev. Lett. 64, 788 (1990).

${ }^{8}$ O. Bohigas, A. M. Lane, and J. Martorell, Phys. Rep. 51, 267 (1979).

${ }^{9}$ F. E. Bertrand, Nucl. Phys. A 354, 129c (1981).

${ }^{10}$ L1. Serra, F. Garcias, M. Barranco, J. Navarro, and Nguyen Van Giai, Z. Phys. D 20, 277 (1991).

${ }^{11}$ Ll. Serra, J. Navarro, M. Barranco, and Nguyen Van Giai, Phys. Rev. Lett. 67, 2311 (1991).

${ }^{12}$ S. Weisgerber and P.-G. Reinhard, Z. Phys. D 23, 275 (1992).

${ }^{13}$ W. Ekardt, Phys. Rev. B 32, 1961 (1985).

${ }^{14}$ L1. Serra, F. Garcias, M. Barranco, J. Navarro, C. Balbás, and A. Mañanes, Phys. Rev. B 39, 8247 (1989).

${ }^{15}$ D. C. Glattli, E. Y. Andrei, G. Deville, J. Poitrenaud, and F. I. B. Williams, Phys. Rev. Lett. 54, 1710 (1985).

${ }^{16}$ D. B. Mast, A. J. Dahm, and A. L. Fetter, Phys. Rev. Lett. 54, 1706 (1985).

${ }^{17}$ V. B. Sandomirskii, V. A. Volkov, G. R. Aizin, and S. Mikhoilov, Electrochim. Acta 34, 3 (1989).

${ }^{18}$ V. Shikin, S. Nazin, D. Heitmann, and T. Demel, Phys. Rev. B 43, 11903 (1991).

${ }^{19}$ D. A. Broido, K. Kempa, and P. Bakshi, Phys. Rev. B 42, 11400 (1990).
${ }^{20}$ V. Gudmundsson and R. R. Gerhardts, Phys. Rev. B 43, 12098 (1991).

${ }^{21}$ V. Gudmundsson and J. J. Palacios, Phys. Rev. B 52, 11266 (1995).

${ }^{22}$ G. Vignale and M. Rasolt, Phys. Rev. Lett. 59, 2360 (1987); Phys. Rev. B 37, 10685 (1988).

${ }^{23}$ M. Ferconi and G. Vignale, Phys. Rev. B 50, 14722 (1994).

${ }^{24}$ P. A. Maksym and T. Chakraborty, Phys. Rev. Lett. 65, 108 (1990).

${ }^{25}$ P. Bakshi, D. A. Broido, and K. Kempa, Phys. Rev. B 42, 7416 (1990).

${ }^{26}$ R. P. Feynmann, Statistical Mechanics (Benjamin, Reading, MA, 1972).

${ }^{27}$ U. Merkt, Physica B 189, 165 (1993).

${ }^{28}$ W. Kohn, Phys. Rev. 123, 1242 (1961).

${ }^{29}$ I. S. Gradshteyn and I. M. Ryzhik, Table of Integrals, Series and Products (Academic, New York, 1980).

${ }^{30}$ P. Gleissl, M. Brack, J. Meyer, and P. Quentin, Ann. Phys. (N.Y.) 197, 205 (1990).

${ }^{31}$ L1. Serra, F. Garcias, M. Barranco, N. Barberán, and J. Navarro, Phys. Rev. B 41, 3434 (1990).

${ }^{32}$ S. Giovanazzi, L. Pitaevskii, and S. Stringari, Phys. Rev. Lett. 72, 3230 (1994).

${ }^{33}$ M. Barranco, A. Polls, and J. Martorell, Nucl. Phys. A 444, 445 (1985).

${ }^{34}$ M. Ferconi, M. R. Geller, and G. Vignale, Phys. Rev. B 52, 16 357 (1995).

${ }^{35}$ C. Guet and W. R. Johnson, Phys. Rev. B 45, 11283 (1992).

${ }^{36}$ B. Godman and A. Sjölander, Phys. Rev. B 8, 200 (1973).

${ }^{37}$ A. K. Rajagopal and G. S. Grest, Phys. Rev. B 9, 268 (1974). 